\title{
G-FANO THREEFOLDS, II
}

\author{
YURI PROKHOROV
}

\begin{abstract}
We classify Fano threefolds with only Gorenstein terminal singularities and Picard number greater than 1 satisfying an additional assumption that the $G$-invariant part of the Weil divisor class group is of rank 1 with respect to an action of some group $G$.
\end{abstract}

\section{InTRODUCTION.}

This work is a sequel to Pro10.

1.1. Let $X$ be a Fano threefold with at worst terminal Gorenstein singularities defined over a field $\mathbb{k}$ of characteristic 0 . Assume that a group $G$ acts on $\bar{X}:=X \otimes_{\mathbb{k}} \overline{\mathbb{k}}$, where $\overline{\mathbb{k}}$ is the algebraic closure of $\mathbb{k}$. Moreover, we assume that $X, G$ and $\mathbb{k}$ satisfy one of the following two conditions.

(a) Algebraic case. $G$ is the Galois group of $\overline{\mathbb{k}}$ over $\mathbb{k}$ acting on $\bar{X}$ through the second factor. The action of $G$ on $X$ is trivial.

(b) Geometric case. The field $\mathbb{k}$ is algebraically closed, $G$ is a finite group, and the action of $G$ on $X$ is given by a homomorphism $G \rightarrow \operatorname{Aut}_{\mathbb{k}}(X)$.

We say that $X$ is a $G$-Fano threefold if $X$ has at worst terminal Gorenstein singularities, $-K_{X}$ is ample, and

$$
\mathrm{Cl}(X)^{G} \text { is a subgroup of rank } 1 \text { containing }-K_{X} \text {. }
$$

where $\operatorname{Cl}(X)$ is the Weil divisor class group of $X$. We refer to the introduction in [Pro09], [Pro10], and [Pro10b] for the motivation behind this definition.

In this paper we give a classification of one class of Gorenstein $G$ Fano threefolds. More general we assume that the group $G$ acts only on the Picard lattice $\operatorname{Pic}(X)$ in an appropriate way (so we do not assume that $G$ acts on the variety itself).

1991 Mathematics Subject Classification. 14E07, 14E30, 14J30, 14J45, 14J50.

Key words and phrases. Fano variety, del Pezzo variety, terminal singularity.

The work was partially supported by the grant RFBR Nos. 11-01-00336-a, 1101-92613-KO_a, Leading Scientific Schools No. 4713.2010.1, and AG Laboratory HSE RF government grant ag. 11.G34.31.0023. 
1.2. Theorem. Let $X$ be a smooth Fano threefold over an algebraically closed field of characteristic 0. Assume that $\rho(X)>1$ and a finite group $G$ acts on $\operatorname{Pic}(X)$ preserving the intersection form and the class $c_{1}=\left[-K_{X}\right]$. Furthermore, assume that $\operatorname{Pic}(X)^{G} \simeq \mathbb{Z}$. Then $X$ is one of the varieties in the table below.

\begin{tabular}{|c|c|c|c|}
\hline No. & $\rho(X)$ & $-K_{X}^{3}$ & $X$ \\
\hline$(1.2 .1)$ & 2 & 12 & $\begin{array}{l}\text { Let } Z_{6} \subset \mathbb{P}^{8} \text { be the image of the Segre em- } \\
\text { bedding of } \mathbb{P}^{2} \times \mathbb{P}^{2} \text { and let } Y_{6} \subset \mathbb{P}^{9} \text { be the } \\
\text { projective cone over } Y_{6} \text {. Then } X \text { is an inter- } \\
\text { section of } Y_{6} \text { with a hyperplane and a quadric }\end{array}$ \\
\hline$(1.2 .2)$ & 2 & 20 & $\begin{array}{l}X \text { is an intersection of three divisors of bide- } \\
\text { gree }(1,1) \text { in } \mathbb{P}^{3} \times \mathbb{P}^{3}\end{array}$ \\
\hline$(1.2 .3)$ & 2 & 28 & $\begin{array}{l}\text { Let } \sigma: Y^{\prime} \rightarrow \mathbb{P}^{5} \text { be the blowup with center a } \\
\text { Veronese surface. Then } X \text { is an intersection } \\
\text { of } D_{1} \in\left|\sigma^{*} \mathscr{O}_{\mathbb{P}^{5}}(1)\right| \text { and } D_{2} \in\left|\sigma^{*} \mathscr{O}_{\mathbb{P}^{5}}(2)-E\right| \\
\text { where } E \text { is the exceptional divisor }\end{array}$ \\
\hline$(1.2 .4)$ & 2 & 48 & $V_{6} \subset \mathbb{P}^{2} \times \mathbb{P}^{2}$, a divisor of bidegree $(1,1)$ \\
\hline$(1.2 .5)$ & 3 & 12 & $\begin{array}{l}X \text { is a double cover of } \mathbb{P}^{1} \times \mathbb{P}^{1} \times \mathbb{P}^{1} \text { whose } \\
\text { branch locus is a member of }\left|-K_{\mathbb{P}^{1} \times \mathbb{P}^{1} \times \mathbb{P}^{1}}\right|\end{array}$ \\
\hline$(1.2 .6)$ & 3 & 30 & $\begin{array}{l}X \text { is an intersection of divisors of tridegrees } \\
(0,1,1),(1,0,1),(1,1,0) \text { in } \mathbb{P}^{2} \times \mathbb{P}^{2} \times \mathbb{P}^{2}\end{array}$ \\
\hline$(1.2 .7)$ & 3 & 48 & $X=\mathbb{P}^{1} \times \mathbb{P}^{1} \times \mathbb{P}^{1}$ \\
\hline$(1.2 .8)$ & 4 & 24 & $\begin{array}{l}X \subset \mathbb{P}^{1} \times \mathbb{P}^{1} \times \mathbb{P}^{1} \times \mathbb{P}^{1} \text { is a divisor of multi- } \\
\text { degree }(1,1,1,1)\end{array}$ \\
\hline
\end{tabular}

1.2.9. Remark. It is easy to see that all the Fano varieties in the table, except for (1.2.1) and (1.2.5), are rational. Varieties (1.2.1) and (1.2.5) are not rational (see [AB92]).

Theorem 1.2 will be proved in Sect. 5. In Sect. \$2 we give several examples. Sections 3 and 4 are preliminary. Finally, in Sect. 6 we investigate singular $G$-Fano threefolds with $\rho(X)>1$. 
Notation. We work over an algebraically closed field $\mathbb{k}$ of characteristic 0. By $c_{1} \in \operatorname{Pic}(X)$ we denote the anticanonical class $-K_{X}$ of $X$, $\mathrm{Cl}(X)$ denotes the Weil divisor class group.

Acknowledgments. I would like to thank Sergei Galkin for useful comments.

\section{EXAMPLES AND REMARKS}

2.1. Remark. By construction, in all cases except for (1.2.5) and (1.2.7) the variety $X$ is a complete intersection of certain Cartier divisors in some higher-dimensional variety $Y$, where $Y=Y_{6}$ in the case (1.2.1),$Y=\mathbb{P}^{3} \times \mathbb{P}^{3}$ in the case (1.2.2), $Y=Y^{\prime}$ in the case (1.2.3), $Y=\mathbb{P}^{2} \times \mathbb{P}^{2}$ in the case (1.2.4), $Y=\mathbb{P}^{2} \times \mathbb{P}^{2} \times \mathbb{P}^{2}$ in the case (1.2.6), and $Y=\mathbb{P}^{1} \times \mathbb{P}^{1} \times \mathbb{P}^{1} \times \mathbb{P}^{1}$ in the case (1.2.8). The embedding $X \subset Y$ is canonical. This means, in particular, that automorphisms of $X$ are induced by those of $Y$.

Below we give some comments on the varieties in the table.

2.2. Case (1.2.1). The variety $X$ can be one of the following two types:

a) $X \subset \mathbb{P}^{2} \times \mathbb{P}^{2}$ is a divisor of bidegree $(2,2)$, or

b) $X$ is a double cover of $V_{6} \subset \mathbb{P}^{2} \times \mathbb{P}^{2}$ (see (1.2.4) ), whose branch locus is a member of $\left|-K_{V_{6}}\right|$.

2.3. Case (1.2.2). The variety $X$ is the blow-up of $\mathbb{P}^{3}$ along a curve of degree 6 and genus 3 which is an intersection of cubics. Indeed, two projections $f_{i}: X \rightarrow \mathbb{P}^{3}$ are blowups of some smooth curve $C \subset \mathbb{P}^{3}$. Easy computations show that $C$ must satisfy the above conditions.

Conversely, let $X$ be the blow-up of $\mathbb{P}^{3}$ along a curve of degree 6 and genus 3 which is an intersection of cubics. By [MM83, §5] on $X$ there are two blowup structures $f_{i}: X \rightarrow \mathbb{P}^{3}$ of this type. These two contractions induce a map $f=f_{1} \times f_{2}: X \rightarrow \mathbb{P}^{3} \times \mathbb{P}^{3}$ which must be finite and birational onto its image. Consider the composition $f^{\prime}: X \stackrel{f}{\rightarrow} \mathbb{P}^{3} \times \mathbb{P}^{3} \stackrel{s}{\rightarrow} \mathbb{P}^{15}$, where $s$ is the Segre embedding. Let $M_{i}:=f_{i}^{*} \mathscr{O}_{\mathbb{P}^{3}}(1)$. Then $-K_{X}=M_{1}+M_{2}$. By Riemann-Roch $\operatorname{dim}\left|-K_{X}\right|=12$. Therefore, $f^{\prime}(X)$ is contained into a subspace of codimension 3, i.e., $f^{\prime}(X) \subset s\left(\mathbb{P}^{3} \times \mathbb{P}^{3}\right) \cap \mathbb{P}^{12}$. Since $\operatorname{deg} f^{\prime}(X)=20=$ $\operatorname{deg} s\left(\mathbb{P}^{3} \times \mathbb{P}^{3}\right) \cap \mathbb{P}^{12}$, we have $f(X) \simeq f^{\prime}(X)=s\left(\mathbb{P}^{3} \times \mathbb{P}^{3}\right) \cap \mathbb{P}^{12}$. By subadjunction, $K_{X}=f^{*} K_{f(X)}-B$, where $B$ is an effective divisor defined by the conductor ideal. Since, $\omega_{f(X)}=\mathscr{O}_{f(X)}(-1,-1)$, we have $B=0$. Therefore, $f(X)$ is normal and $f(X) \simeq X$. 
2.3.1. Example (cf. Kat87]). Let $\left(a_{i, j}\right),\left(b_{i, j}\right),\left(c_{i, j}\right)$ be symmetric $4 \times 4$-matrices and let $X \subset \mathbb{P}_{x_{1} \ldots, x_{4}}^{3} \times \mathbb{P}_{y_{1} \ldots, y_{4}}^{3}$ is given by the equations

$$
\sum a_{i, j} x_{i} y_{j}=\sum b_{i, j} x_{i} y_{j}=\sum c_{i, j} x_{i} y_{j}=0 .
$$

If $\left(a_{i, j}\right),\left(b_{i, j}\right),\left(c_{i, j}\right)$ are taken sufficiently general, then $X$ is a smooth Fano threefold of type (1.2.2). The group $\boldsymbol{\mu}_{2}$ acts on $X$ by $x_{i} \mapsto y_{i}$. The induced birational involution $\mathbb{P}^{3} \rightarrow \mathbb{P}^{3}$ is cubo-cubic, it is given by the linear system of cubics passing through the center of blowup $C \subset \mathbb{P}^{3}$. The exceptional divisor is sweep out by trisecants of $C$ and it is a surface $F \subset \mathbb{P}^{3}$ of degree 8 with multiplicity 3 along $C$ [Kat87].

2.4. Case (1.2.3). It is easy to show that $Y^{\prime}$ is a Fano fivefold. Linear systems $\left|\mathscr{O}_{\mathbb{P} 5}(1)\right|$ and $\left|\mathscr{O}_{\mathbb{P}^{5}}(2)-E\right|$ define two contractions $h_{i}: Y^{\prime} \rightarrow \mathbb{P}^{5}$ which are blowups of Veronese surfaces $V=V_{4} \subset \mathbb{P}^{5}$ (cf. [CK89]). In this case, $X$ can be realized as the blow-up of a smooth quadric $Q \subset \mathbb{P}^{4}$ along a twisted quartic curve. Indeed, the restriction $f_{1}=\left.h_{1}\right|_{X}$ is a birational map whose image is a quadric $Q=h_{1}\left(D_{1}\right) \cap h_{2}\left(D_{2}\right) \subset \mathbb{P}^{5}$ and moreover $f_{i}$ is the blowup of $Q$ along $V \cap h_{1}\left(D_{1}\right)$, a twisted quartic curve.

2.4.1. Example. Let $C \subset \mathbb{P}^{4}$ be a rational normal curve of degree 4 . The action of the group $\operatorname{Aut}(C)=\mathrm{PGL}_{2}(\mathbb{k})$ naturally extends to $\mathbb{P}^{4}$ so that $\mathbb{P}^{4}=\mathbb{P}\left(S^{4} V\right)$, where $V$ is the standard representation of $\mathrm{GL}_{2}(\mathbb{k})$. The representation of $\mathrm{GL}_{2}(\mathbb{k})$ on $S^{4} V$ is irreducible and can be defined by matrices over $\mathbb{R}$. Therefore, there exists an invariant non-singular quadric $Q \subset \mathbb{P}^{4}$ containing $C$. Let $f_{1}: X \rightarrow Q$ be the blowup of $C$. Then $X$ is a Fano threefold of type (1.2.3) . The $\mathrm{PGL}_{2}(\mathbb{k})$-action lifts to $X$ and the second contraction $f_{2}: X \rightarrow Q$ is also $\mathrm{PGL}_{2}(\mathbb{k})$ equivariant. Let $C^{\prime} \subset Q$ be the center of the blowup $f_{2}$. Clearly, the pairs $(Q, C)$ and $\left(Q, C^{\prime}\right)$ are $\mathrm{SL}_{2}(\mathbb{k})$-isomorphic, i.e. there exists an automorphism $\gamma: Q \rightarrow Q$ such that $\gamma(C)=C^{\prime}$ (because $C$ is the only one-dimensional orbit on $Q$ ). Then $f_{2} \circ \gamma \circ f_{1}^{-1}$ is an involution on $X$.

2.5. Case (1.2.6). Here $X$ is the blow-up of $V_{6} \subset \mathbb{P}^{2} \times \mathbb{P}^{2}$ (see (1.2.4)), along a curve $C$ of bidegree $(2,2)$ such that the composition $C \hookrightarrow$ $V_{6} \hookrightarrow \mathbb{P}^{2} \times \mathbb{P}^{2} \stackrel{p_{i}}{\rightarrow} \mathbb{P}^{2}$ is an embedding for each projection $p_{i}, i=1,2$. Indeed, there are three projections $\pi:=X \rightarrow \mathbb{P}^{2} \times \mathbb{P}^{2}$. The image of each $\pi_{i}$ is contained into a divisor $V \subset \mathbb{P}^{2} \times \mathbb{P}^{2}$ of type $(1,1)$ and $\pi_{i}$ is birational onto its image. Hence $\pi_{i}$ passes through a birational extremal contraction $X \rightarrow V^{\prime} \rightarrow V$. By [MM82, Table 3] we have $V^{\prime} \simeq V, V$ is smooth and $\pi_{i}$ is a blowup of a curve as above.

2.5.1. Example (cf. Nak89]). Let $\Gamma \subset \mathbb{P}^{2}$ is a non-degenerate conic and let $\Gamma^{*} \subset \mathbb{P}^{2 *}$ be its dual, the conic formed by lines that are tangent 
to $\Gamma$. Consider the incidence curve

$$
C=\left\{(P, L) \in \Gamma \times \Gamma^{*} \subset \mathbb{P}^{2} \times \mathbb{P}^{2 *} \mid L \text { is tangent to } \Gamma \text { at } P\right\} .
$$

Then $C$ is contained into the flag variety $\mathrm{Fl}\left(\mathbb{P}^{2}\right)=V_{6}$ and satisfies conditions of (1.2.6). The action $\operatorname{Aut}(\Gamma)=\mathrm{PGL}_{2}(\mathbb{k})$ extends to $V_{6}=$ $\mathrm{Fl}\left(\mathbb{P}^{2}\right)$. Orbits of $\mathrm{PGL}_{2}(\mathbb{k})$ on $V_{6}$ are described as follows:

- $C \simeq \mathbb{P}^{1} \simeq \mathrm{SL}_{2}(\mathbb{k}) / B$, where $B$ is a Borel subgroup,

- $D^{\prime}$ and $D^{\prime \prime}$, where $\bar{D}^{\prime}:=D^{\prime} \cup C$ and $\bar{D}^{\prime \prime}:=D^{\prime \prime} \cup C$ are complete surfaces isomorphic to $\mathbb{P}^{1} \times \mathbb{P}^{1}$ with the diagonal action of $\mathrm{PGL}_{2}(\mathbb{k})$,

- an open orbit $U \simeq \mathrm{SL}_{2}(\mathbb{k}) / Q_{8}$, where $Q_{8}$ is the binary quaternion group of order 8 .

Let $f: X \rightarrow V_{6}$ be the blowup of $C$. Then $X$ is a Fano threefold of type (1.2.6) admitting a $\mathrm{PGL}_{2}(\mathbb{k})$-action. There are two more $\mathrm{PGL}_{2}(\mathbb{k})$-equivariant contractions $f^{\prime}, f^{\prime \prime}: X \rightarrow V_{6}$ contracting proper transforms of $\bar{D}^{\prime}$ (resp. $\left.\bar{D}^{\prime \prime}\right)$ to curves $C^{\prime} \subset V_{6}$ (resp. $C^{\prime \prime} \subset V_{6}$ ) of bidegree $(2,2)$. The pairs $\left(V_{6}, C\right),\left(V_{6}, C^{\prime}\right)$, and $\left(V_{6}, C^{\prime \prime}\right)$, are isomorphic. These isomorphisms induce an action of the symmetric group $\mathfrak{S}_{3}$ on $X$.

2.6. Case (1.2.8). The variety $X$ is isomorphic to the blowup of $\mathbb{P}^{1} \times \mathbb{P}^{1} \times \mathbb{P}^{1}$ along an elliptic curve which is an intersection of two members of $\left|-\frac{1}{2} K_{\mathbb{P}^{1} \times \mathbb{P}^{1} \times \mathbb{P}^{1}}\right|$. Similar to 2.3 .1 one can easily construct examples of symmetric varieties of this type (admitting a $G$-structure).

\section{Action on the Picard lattice.}

3.1. Lemma. Let $V=\mathbb{Q}^{N}$ and let $\Phi: G \hookrightarrow \operatorname{GL}(V)$ be a faithful representation of a finite group $G$. Identify $G$ with its image $\Phi(G) \subset$ $\mathrm{GL}(V)$ and assume that $V^{G}=0$. Then there is a subgroup $G_{0} \subset G$ such that $V^{G_{0}}=0$ and

$$
\left|G_{0}\right|= \begin{cases}2 & \text { if } N=1, \\ 2 \text { or } 3 & \text { if } N=2, \\ 2,4, \text { or } 6 & \text { if } N=3, \\ 5,9, \text { or divides } 3 \cdot 2^{7} & \text { if } N=4 .\end{cases}
$$

Proof. Cases $N \leq 2$ are trivial. Consider the case $N=3$. According to Minkowski's bound (see e.g. [Ser07]) the order of any subgroup $G \subset$ $\mathrm{GL}_{3}(\mathbb{Q})$ divides 48. Assume that there is an element $\tau \in G$ such that $V^{\langle\tau\rangle}=0$. Let $\mu(t)$ be the minimal polynomial of $\tau$. Then $\operatorname{deg} \mu \leq 3, \mu$ is a product of cyclotomic polynomials $\phi_{k}(t)$, and $\mu(1) \neq 0$. Moreover, in some basis, $\tau$ is given by an orthogonal matrix, so $\mu(-1)=0$ and $\phi_{2} \mid \mu$. Hence, there are the following possibilities: $\mu=\phi_{2}, \phi_{2} \phi_{4}, \phi_{2} \phi_{3}$, or $\phi_{2} \phi_{6}$. Thus we can take $G_{0}=\langle\tau\rangle$. 
Now we assume that for any element $\tau \in G$ we have $V^{\langle\tau\rangle} \neq 0$. In particular, $G$ is not a cyclic group. We also may assume that $|G| \geq 8$. If $G$ contains a subgroup $G_{1} \subset G$ isomorphic to $\boldsymbol{\mu}_{2} \times \boldsymbol{\mu}_{2}$, then either $G_{1} \subset \mathrm{SL}(V)$ or $G_{1} \ni-\mathrm{id}$. In the latter case we can take $G_{0}=\langle-\mathrm{id}\rangle$ and in the former case we can take $G_{0}=G_{1}$. Note that $\operatorname{GL}(V)$ contains no elements of order 8 and the quaternion group has no real faithful representations of dimension $\leq 3$. Thus we may assume that the order of $G$ is not divisible by 8 . We get only one possibility: Sylow 2-subgroups of $G$ are cyclic of order 4 and $G$ is of order 12. Let $G_{1} \subset G$ be a Sylow 2-subgroup and let $\tau$ be its generator. We may assume that $G_{1} \subset \operatorname{SL}(V)$ (otherwise we can take $G_{0}=G_{1}$ ). Then $G \subset \operatorname{SL}(V)$. If $G$ has an element $\xi$ of order 6 , then $\xi$ generates the desired subgroup $G_{0}$. Otherwise the representation $\Phi: G \hookrightarrow \mathrm{SL}(V)$ is irreducible. Then we must have $G \simeq \mathfrak{A}_{4}$ and $G_{1}$ is not cyclic, a contradiction.

Consider the case $N=4$. Again according to Minkowski's bound (see e.g. Ser07]) the order of any subgroup $G \subset \mathrm{GL}_{4}(\mathbb{Q})$ divides $2^{7} \cdot 3^{2} \cdot 5$. Again if there is an element $\tau \in G$ such that $V^{\langle\tau\rangle}=0$, then its minimal polynomial $\mu(t)$ satisfies the following conditions: $\operatorname{deg} \mu \leq 4$, $\mu$ is a product of cyclotomic polynomials $\phi_{k}(t)$, and $\mu(1) \neq 0$. In this case, we have either $\mu=\phi_{5}, \phi_{8}, \phi_{10}, \phi_{12}$, or $\mu$ divides $\phi_{2} \phi_{3} \phi_{4} \phi_{6}$. Thus we can take $G_{0}=\langle\tau\rangle$.

Now we assume that for any element $\tau \in G$ we have $V^{\langle\tau\rangle} \neq 0$. Then $G$ contains no elements of order 5,9, and 8. In particular, the order of $G$ divides $2^{7} \cdot 3^{2}$. Let $G_{1} \subset G$ be a Sylow 3 -subgroup. By the above, we may assume that $G_{1} \simeq \boldsymbol{\mu}_{3} \times \boldsymbol{\mu}_{3}$. In this case $V^{G_{1}}=\{0\}$ and we can take $G_{0}=G_{1}$.

3.1.1. Corollary. In notation of Theorem 1.2 there is a subgroup $G_{0} \subset G$ of order $N$ such that $\rho(X)^{G_{0}}=1$, where

$$
N= \begin{cases}2 & \text { if } \rho(X)=2, \\ 2 \text { or } 3 & \text { if } \rho(X)=3, \\ 2,4 \text { or } 6 & \text { if } \rho(X)=4, \\ 5,9, \text { or divides } 3 \cdot 2^{7} & \text { if } \rho(X)=5 .\end{cases}
$$

Proof. Let $V$ be the orthogonal complement to $c_{1}$ in $\operatorname{Pic}_{\mathbb{Q}}(X):=$ $\operatorname{Pic}(X) \otimes \mathbb{Q}$. The faithful representation $G \hookrightarrow \mathrm{GL}(V)$ satisfies the condition $V^{G}=0$. Then we can apply Lemma 3.1.

\section{Fano Conic Bundles And Del Pezzo fibrations.}

4.1. Definition. Let $X$ be a smooth threefold. A morphism $f$ : $X \rightarrow Z$ onto a smooth surface is a conic bundle if every (scheme) fiber is isomorphic to a conic in $\mathbb{P}^{2}$. A morphism $f: X \rightarrow Z$ onto a 
smooth curve is a del Pezzo bundle if $f$ has connected fibers and $-K_{X}$ is $f$-ample. (In this case a general fiber of $f$ is a del Pezzo surface.)

4.1.1. Remark. Let $X$ be a smooth Fano threefold and let $f: X \rightarrow Z$ be a surjective morphism with connected fibers.

(i) If $Z$ is a smooth curve, then $f$ is a del Pezzo bundle and $Z \simeq \mathbb{P}^{1}$.

(ii) If $Z$ is a smooth surface and $f$ is equidimensional, then $f$ is a conic bundle and $Z$ is rational.

4.1.2. Proposition ([MM86, §4]). Let $X$ be a Fano threefold. Assume that $X$ has a conic bundle structure $f: X \rightarrow Z$. Then

(i) $Z$ is a del Pezzo surface.

(ii) The discriminant curve $\Delta \subset Z$ is a curve with at worst ordinary double points (or empty).

(iii) $\rho(X / Z)=1$ if and only if for any irreducible curve $C \subset Z$ its preimage $f^{-1}(C)$ is also irreducible.

(iv) If $C \subset Z$ is a an irreducible curve such that $f^{-1}(C)$ is reducible, then $C$ is a smooth connected component of $\Delta$.

(v) $h^{1,2}(X)=\rho(X)-\rho(Z)+p_{a}(\Delta)-2$.

4.1.3. Corollary. If in the assumptions of Proposition $4.1 .2 \rho(Z) \geq$ 2, then $X$ has a del Pezzo bundle structure.

4.2. Assumption. ¿From now on we assume that $X$ is a smooth Fano threefold satisfying assumptions of Theorem 1.2. By [Pro10] we may assume that the Fano index of $X$ is equal to 1 (otherwise $X$ is of type (1.2.4) or (1.2.7)). Thus from now on we assume that $\operatorname{Pic}(X)^{G}$ is generated by $-K_{X}$. We also assume that $G$ is the smallest group satisfying condition (1.1.1) (cf. Corollary 3.1.1).

4.3. Proposition. Assume that $X$ has a conic bundle structure $f$ : $X \rightarrow Z$ over $Z=\mathbb{P}^{2}$. Then $X$ is of type (1.2.1) or (1.2.6).

Proof. According to [MM83, Prop. 6.3] $\rho(X) \leq 3$. By Corollary 3.1.1 we may assume that $|G| \leq 3$. Let $L \subset \mathbb{P}^{2}$ be a line, let $F:=f^{-1}(L)$, and let $\Delta \subset \mathbb{P}^{2}$ be the discriminant curve. Let $F_{1}, \ldots, F_{n}$ be the $G$ orbit of the class of $F$ in $\operatorname{Pic}(X)$, where $n=2$ or 3 . Write $\sum F_{i}=a c_{1}$ for some $a \in \mathbb{Z}$. Take the line $L$ to be sufficiently general, so the surface $F$ is smooth. Then we have

$$
\begin{aligned}
n(12-\operatorname{deg} \Delta) & =n K_{F}^{2}+4 n=n\left(K_{X}+F\right)^{2} \cdot F- \\
& -2 n c_{1} \cdot F^{2}=n c_{1}^{2} \cdot F=c_{1}^{2} \cdot \sum F_{i}=a c_{1}^{3}>0 .
\end{aligned}
$$

In particular, $\operatorname{deg} \Delta<12$. 
First assume that $n=2$. As above $F_{1}+F_{2}=a c_{1}$ and so

$$
a^{2} c_{1}^{3}=\frac{1}{a}\left(F_{1}+F_{2}\right)^{3}=\frac{6}{a} F_{1}^{2} \cdot F_{2}=\frac{6}{a} F_{1}^{2} \cdot\left(F_{1}+F_{2}\right)=6 c_{1} \cdot F^{2}=12 .
$$

Since $c_{1}^{3}$ is even, there is only one possibility: $a=1, c_{1}^{3}=12$, and $\operatorname{deg} \Delta=6$. By Proposition 4.1.2 $h^{1,2}(X)=\rho(X)+7$. Then from tables in [MM82] we get the case (1.2.1).

Assume that $n=3$. Then $\rho(X)=3$ and $\Delta \neq \emptyset$. From (4.3.1) we see $3(12-\operatorname{deg} \Delta)=a c_{1}^{3}$. So, $a c_{1}^{3} \leq 33$.

If $a \geq 2$, then from [MM82, Table 3] we get $a=2$. Then $c_{1}^{3}=$ $18-\frac{3}{2} \operatorname{deg} \Delta$. Since $c_{1}^{3}$ is even, $c_{1}^{3}=12$ and $\operatorname{deg} \Delta=4$. But in this case, $h^{1,2}(X)=p_{a}(\Delta)=3$. This contradicts [MM82, Table 3]. Therefore, $a=1$ and $c_{1}^{3}=3(12-\operatorname{deg} \Delta)$. In particular, $\operatorname{deg} \Delta$ is even and $c_{1}^{3} \leq 30$. Moreover, if $c_{1}^{3}=30$, then by [MM82, Table 3] we get the case (1.2.6). Thus we may assume that $c_{1}^{3}<30$. There are the following possibilities:

$$
\begin{aligned}
& -\operatorname{deg} \Delta=4, c_{1}^{3}=24, h^{1,2}(X)=p_{a}(\Delta)=3, \\
& -\operatorname{deg} \Delta=6, c_{1}^{3}=18, h^{1,2}(X)=p_{a}(\Delta)=10 \\
& -\operatorname{deg} \Delta=8, c_{1}^{3}=12, h^{1,2}(X)=p_{a}(\Delta)=21
\end{aligned}
$$

All these cases are impossible by the classification [MM82, Table 3].

4.4. Lemma. Assume that $X$ has a del Pezzo bundle structure $f$ : $X \rightarrow \mathbb{P}^{1}$ and let $F$ be a general fiber. Let $F_{1}, \ldots, F_{n}$ be the $G$-orbit of the class of $F$ in $\operatorname{Pic}(X)$. Write $\sum F_{i}=a c_{1}$ for some $a \in \mathbb{Z}$. Then

(i) $9 n \geq n K_{F}^{2}=a c_{1}^{3}, \quad a>0$,

(ii) $n>2,|G|>2$, and $\rho(X) \geq 3$,

(iii) $a c_{1}^{3}$ is divisible by 3 ,

(iv) $a K_{F}^{2}$ is even,

(v) if $n=3$, then $\rho(X)=3$ and either

(a) $X$ is of type (1.2.5), or

(b) $c_{1}^{3}=24$ and $K_{F}^{2}=8$.

Proof. We have

$$
9 n \geq n K_{F}^{2}=n c_{1}^{2} \cdot F=c_{1}^{2} \cdot \sum F_{i}=a c_{1}^{3} .
$$

This proves (i). Further,

$$
6 \sum_{i<j<k} F_{i} \cdot F_{j} \cdot F_{k}=\left(\sum F_{i}\right)^{3}=a^{3} c_{1}^{3}>0 .
$$

In particular, $a c_{1}^{3}$ is divisible by 3 and $n>2$. Further,

$$
2 F \cdot \sum_{1<i<j} F_{i} \cdot F_{j}=F \cdot\left(\sum F_{i}\right)^{2}=a^{2} F \cdot c_{1}^{2}=a^{2} K_{F}^{2} .
$$


Hence, $a^{2} K_{F}^{2}$ is even.

Finally, let $n=3$. Then $\rho(X) \geq 3$. If $c_{1}^{3} \leq 12$, then by MM82, Tables 3-5] we are in the case (1.2.5). Thus we may assume that $c_{1}^{3}>12$. Since $c_{1}^{3}$ is even, $a c_{1}^{3} \leq 24$. Therefore, $a=1$. Let $\Gamma:=\left.F_{2}\right|_{F}$. Further,

$$
-K_{F} \cdot \Gamma=c_{1} \cdot F_{2} \cdot F_{1}=F_{1} \cdot F_{2} \cdot F_{3}=\frac{1}{6}\left(F_{1}+F_{2}+F_{3}\right)^{3}=\frac{c_{1}^{3}}{6} .
$$

Since $\Gamma^{2}=F_{2}^{2} \cdot F=0$, by Riemann-Roch $K_{F} \cdot \Gamma$ is even. Hence $c_{1}^{3}$ is divisible by 12 . We get $c_{1}^{3}=24$ and $K_{F}^{2}=8$. If $\rho(X) \geq 4$, then by [MM82, Tables 4-5] we have $\rho(X)=4$ and $X$ is of type (1.2.8). But in this case, $X$ has a del Pezzo bundle structure of degree 6 , a contradiction.

4.5. Lemma. $X \not z Z \times \mathbb{P}^{1}$, where $Z$ is a smooth surface.

Proof. Clearly, $Z$ is a del Pezzo surface of degree $10-\rho(Z)$. Let $F$ be a fiber of the projection $X=Z \times \mathbb{P}^{1} \rightarrow \mathbb{P}^{1}$. Take an element $\tau \in G$ so that ${ }^{\tau} F$ is not proportional to $F$. Then ${ }^{\tau} F \sim \alpha F+f^{*} L$ for some $0 \neq L \in \operatorname{Pic}(Z)$ and $\alpha \in \mathbb{Z}$. Since $F^{2} \equiv 0$, we have

$$
0={ }^{\tau} F^{2} \cdot F=f^{*} L^{2} \cdot F .
$$

Hence, $L^{2}=0$ and $2 \alpha F \cdot f^{*} L \equiv{ }^{\tau} F^{2} \equiv 0$. So, $\alpha=0$ and ${ }^{\tau} F=f^{*} L$. Further, by Riemann-Roch $K_{Z} \cdot L$ is even and

$$
K_{Z}^{2}=c_{1}^{2} \cdot F=c_{1}^{2} \cdot{ }^{\tau} F=c_{1}^{2} \cdot f^{*} L=\left(2 F-f^{*} K_{Z}\right)^{2} \cdot f^{*} L=-4 K_{Z} \cdot L .
$$

Therefore, $K_{Z}^{2}=8$ and $\rho(X)=3$. This contradicts Lemma 4.4.

\section{Proof of Theorem 1.2}

Recall our assumption that $\operatorname{Pic}(X)^{G}$ is generated by $c_{1}$ and that $G$ is the smallest group satisfying condition (1.1.1).

5.1. Consider the case $\rho(X)=2$. Denote the generator of $G \simeq \boldsymbol{\mu}_{2}$ by $\tau$. Let $R_{1}$ and $R_{2}$ be extremal rays of the Mori cone $\overline{\mathrm{NE}}(X) \subset$ $\mathrm{N}_{1}(X) \simeq \mathbb{R}^{2}$. Let $f_{i}: X \rightarrow X_{i}$ be the contraction of $R_{i}$, let $A_{i}$ be the ample generator of $\operatorname{Pic}\left(X_{i}\right) \simeq \mathbb{Z}$, and let $M_{i}:=f_{i}^{*} A_{i}$. By Proposition 4.3 and Lemma 4.4 we may assume that both contractions are birational. Let $D_{i}$ be the exceptional divisor of $f_{i}$.

5.1.1. Theorem ([MM83, Th. 5.1]). The group $\operatorname{Pic}(X)$ is generated by $M_{1}$ and $M_{2}$.

By this theorem

$$
-K_{X} \equiv \alpha_{1} M_{1}+\alpha_{2} M_{2}, \quad \alpha_{i} \in \mathbb{Z}
$$


Since $M_{1}+{ }^{\tau} M_{1}$ and $M_{2}+{ }^{\tau} M_{2}$ are invariant divisors, we have

$$
M_{1}+{ }^{\tau} M_{1}=-a_{1} K_{X}, \quad M_{2}+{ }^{\tau} M_{2}=-a_{2} K_{X},
$$

for some $a_{1}, a_{2} \in \mathbb{Z}$. Then

$$
-2 K_{X}=\alpha_{1} M_{1}+\alpha_{2} M_{2}+{ }^{\tau}\left(\alpha_{1} M_{1}+\alpha_{2} M_{2}\right)=-\left(\alpha_{1} a_{1}+\alpha_{2} a_{2}\right) K_{X} .
$$

So, $\alpha_{1} a_{1}+\alpha_{2} a_{2}=2$. On the other hand,

$$
0<2 K_{X}^{2} \cdot M_{i}=K_{X}^{2} \cdot\left(M_{i}+{ }^{\tau} M_{i}\right)=a_{i} c_{1}^{3} .
$$

Therefore, $a_{i}>0$. This gives us $a_{i}=\alpha_{i}=1$, i.e.,

$$
{ }^{\tau} M_{1}=M_{2}, \quad-K_{X}=M_{1}+M_{2} .
$$

Further, $M_{1}^{2} \cdot D_{1}=M_{2}^{2} \cdot D_{2}=0$ and $M_{2}^{2} \cdot{ }^{\tau} D_{1}={ }^{\tau} M_{1}^{2} \cdot{ }^{\tau} D_{1}=0$. Hence, $D_{2}$ and ${ }^{\tau} D_{1}$ are proportional, so ${ }^{\tau} D_{1}=b D_{2}$ for some $b \in \mathbb{Q}$. Since $D_{2} \cdot\left(-K_{X}\right)^{2}>0$ and ${ }^{\tau} D_{1} \cdot\left(-K_{X}\right)^{2}>0, b>0$. Thus

$$
D_{1}+b D_{2}=D_{1}+{ }^{\tau} D_{1}=-c K_{X} \quad \text { for some } c \in \mathbb{Z}_{>0} .
$$

If $f_{1}\left(D_{1}\right)$ is a point, then $M_{1} \cdot D_{1} \equiv M_{2} \cdot D_{2} \equiv 0$, i.e. $f_{2}\left(D_{2}\right)$ is also a point. In this case, $D_{1} \cap D_{2}=\emptyset$. Hence,

$0<c^{2}\left(-K_{X}\right)^{3}=\left(-K_{X}\right) \cdot\left(D_{1}+b D_{2}\right)^{2}=\left(-K_{X}\right) \cdot D_{1}^{2}+b^{2}\left(-K_{X}\right) \cdot D_{2}^{2}<0$, a contradiction.

Therefore, $C_{i}:=f_{i}\left(D_{i}\right)$ are curves. In this case both varieties $X_{i}$ are smooth Fano threefolds. Write $-K_{X_{i}}=r_{i} A_{i}, r_{i}=1,2,3$, or 4 . Since $D_{2}$ and ${ }^{\tau} D_{1}$ are primitive elements of $\operatorname{Pic}(X)$, we have $D_{2}={ }^{\tau} D_{1}$. Then

$$
-K_{X}=-f^{*} K_{X_{1}}-D_{1}=r_{1} M_{1}-D_{1}=r_{1}{ }^{\tau} M_{1}-{ }^{\tau} D_{1}=r_{1} M_{2}-D_{2} .
$$

This shows that ${ }^{\tau} D_{1}=D_{2}$ and $r_{1}=r_{2}$. Put $r:=r_{1}=r_{2}$. Further,

$$
\begin{gathered}
M_{2}^{2} \cdot M_{1}=M_{1}^{2} \cdot M_{2}=M_{1}^{2} \cdot\left((r-1) M_{1}-D_{1}\right)=(r-1) M_{1}^{3}, \\
A_{1} \cdot C_{1}=-M_{1} \cdot D_{1}^{2}=-M_{1} \cdot\left((r-1) M_{1}-M_{2}\right)^{2}=(r-1)(r-2) M_{1}^{3} .
\end{gathered}
$$

Therefore, $r \geq 3$. We get two possibilities: (1.2.2) and (1.2.3).

¿From now on we assume that $\rho(X) \geq 3$.

5.2. Proposition. If in the above assumptions $\rho(X) \geq 3$ and $X$ is not of type (1.2.6), then $X$ has a structure of del Pezzo bundle of degree $\leq 8$.

Proof. If $X$ has a conic bundle structure $f: X \rightarrow Z$, then by Proposition $4.3 Z \not \mathbb{P}^{2}$. Since $Z$ is a smooth rational surface, there exists a surjective morphism $Z \rightarrow \mathbb{P}^{1}$ with connected fibers. The composition map gives a structure of del Pezzo bundle whose fibers $F$ are del Pezzos with $\rho(F)>1$. 
Assume that $X$ has no conic bundle structures. Then by MM83, (9.1), (9.2), (9.6))] the variety $X$ isomorphic to the blow-up of $\mathbb{P}^{3}$ along a disjoint union of a line and a conic. The map $X \rightarrow \mathbb{P}^{3}$ can be decomposed $X \rightarrow X_{1} \rightarrow \mathbb{P}^{3}$, where $X_{1} \rightarrow \mathbb{P}^{3}$ is the blow-up of a line. In this case $X_{1}$ has a structure of a $\mathbb{P}^{2}$-fibration over $\mathbb{P}^{1}$. The composition $X \rightarrow X_{1} \rightarrow \mathbb{P}^{1}$ is the desired map.

5.3. Assume that $\rho(X)=3$. By Proposition $5.2 X$ has a del Pezzo bundle structure $f: X \rightarrow Z$ and by Lemma $4.4 n:=|G|>2$. Let $F$ be a general fiber. By Lemma 4.4 we have $K_{F}^{2}=8$ and $c_{1}^{3}=24$. According to [MM82, Table 3] we have the following possibilities:

5.3.1. Case $7^{\circ} . X$ is a blow-up of $V_{6} \subset \mathbb{P}^{2} \times \mathbb{P}^{2}$ along an elliptic curve $C$ which is an intersection of two members of $\left|-\frac{1}{2} K_{V_{6}}\right|$. Then projection from $C$ gives us a del Pezzo bundle structure $f: X \rightarrow \mathbb{P}^{1}$ of degree 6 . This contradicts $K_{F}^{2}=8$.

5.3.2. Case $8^{\circ} . X$ is a member of the linear system $\mid p_{1}^{*} \sigma^{*} \mathscr{O}(1) \otimes$ $p_{2}^{*} \mathscr{O}(2) \mid$ on $\mathbb{F}_{1} \times \mathbb{P}^{2}$, where $p_{i}$ is the projection and $\sigma: \mathbb{F}_{1} \rightarrow \mathbb{P}^{2}$ is the blowing up. The composition $X \stackrel{p_{1}}{\longrightarrow} \mathbb{F}_{1} \stackrel{q}{\longrightarrow} \mathbb{P}^{1}$, where $q$ is the $\mathbb{P}^{1}$-ruling, is a del Pezzo bundle whose general fiber $F$ is a divisor in $\mathbb{P}^{1} \times \mathbb{P}^{2}$ of bidegree $(1,2)$. Hence, $F$ is a del Pezzo surface of degree 5 . This contradicts $K_{F}^{2}=8$.

5.4. Finally, let $X$ be a Fano threefold with $\rho(X) \geq 4$. By MM82, Tables 4-5] $c_{1}^{3} \geq 24$. Moreover, if $c_{1}^{3}=24$, then $X$ is a divisor of multidegree $(1,1,1,1)$ in $\mathbb{P}^{1} \times \mathbb{P}^{1} \times \mathbb{P}^{1} \times \mathbb{P}^{1}$, i.e. $X$ is of type (1.2.8). Thus from now on we assume that $c_{1}^{3} \geq 26$ and $4 \leq \rho(X) \leq 5$ (see [MM82, Tables 4-5]).

5.5. Assume that $\rho(X)=4$. Then by Corollary 3.1.1 $n=2,3,4$, or 6. According to Lemma 4.4 (i)

$$
48 \geq n K_{F}^{2}=a c_{1}^{3}
$$

and $a c_{1}^{3}$ is divisible by 3 .

Since, $c_{1}^{3} \geq 26$, we have $a=1, n K_{F}^{2} \geq 26$, and $n=4$ or 6 . Further, by Lemma 4.4 (iv) $K_{F}^{2}$ is even. Hence, $c_{1}^{3}$ is divisible by 12 . By [MM82, Table 4] we get the following possibility $7^{\circ}: X$ is the blowup of $V_{6} \subset \mathbb{P}^{2} \times \mathbb{P}^{2}$ of type (1.2.4) along disjoint union of curves of bidegree $(1,0)$ and $(0,1)$. So, there is an embedding $X \subset \mathbb{F}_{1} \times \mathbb{F}_{1}$. The composition

$$
X \hookrightarrow \mathbb{F}_{1} \times \mathbb{F}_{1} \stackrel{p_{1}}{\longrightarrow} \mathbb{F}_{1} \rightarrow \mathbb{P}^{1}
$$

is a del Pezzo bundle whose general fiber is a del Pezzo surface of degree 7. This contradicts (5.5.1). 
5.6. Assume that $\rho(X)=5$. Then by [MM82, Table 5] and Lemma 4.5 we have two possibilities:

5.6.1. Case $1^{\circ}$. Then $c_{1}^{3}=28$ and $X$ can be obtained as a sequence of blow-ups

$$
X \stackrel{h}{\longrightarrow} Y \stackrel{g}{\longrightarrow} Q
$$

where $Q$ is a smooth quadric in $\mathbb{P}^{4}, g$ is the blow-up of a conic $C \subset Q$, and $h$ is the blow-up of three exceptional lines of $g$. As above, the projection $Q \rightarrow \mathbb{P}^{1}$ from $C$ induces a quadric bundle $Y \rightarrow \mathbb{P}^{1}$. The

composition $s: X \stackrel{h}{\longrightarrow} Y \rightarrow \mathbb{P}^{1}$ is a del Pezzo bundle whose general fiber $F$ is the blow-up of three points on the corresponding fiber of $Y \rightarrow \mathbb{P}^{1}$. Hence, $F$ is a del Pezzo surface of degree 5. By Lemma 4.4 (i) we have $5 n=n K_{F}^{2}=28 a$. Since $\left|G_{0}\right|$ is not divisible by 7 , we get a contradiction.

5.6.2. Case $2^{\circ} \cdot c_{1}^{3}=36$ and $X$ can be obtained as a sequence of blow-ups

$$
X \stackrel{h}{\longrightarrow} Y \stackrel{g}{\longrightarrow} \mathbb{P}^{3}
$$

where $g$ is the blow-up of two disjoint lines $L_{1}, L_{2} \subset \mathbb{P}^{3}$ and $h$ is the blow-up of two exceptional lines $\ell, \ell^{\prime} \subset g^{-1}\left(L_{1}\right)$. The projection from $L_{2}$ induces a del Pezzo bundle $s: X \rightarrow \mathbb{P}^{1}$ of degree 8 and projection from $L_{1}$ induces a del Pezzo bundle $r: X \rightarrow \mathbb{P}^{1}$ of degree 6 . Let $F$ be a general fiber of $s$. Then by Lemma 4.4 (i)

$$
8 n=-a K_{X}^{3}=36 a, \quad 2 n=9 a .
$$

Hence 9 divides $n$ and we may assume that $n=9$ and $a=2$. By Lemma 3.1 and our assumption $|G|=9$. Let $F^{\prime}$ be a general fiber of $r$. Again by Lemma 4.4 (i)

$$
6 n^{\prime}=-a^{\prime} K_{X}^{3}=36 a^{\prime}, \quad n^{\prime}=6 a^{\prime} .
$$

On the other hand, $n^{\prime}$ divides $|G|=9$, a contradiction.

This finishes the proof of Theorem 1.2.

\section{Singular CASE}

In this section we consider the case of singular $G$-Fano threefolds with $\rho(X)>1$. On this step we may assume that the ground field is $\mathbb{C}$.

6.1. Theorem ([Nam97]). Let $X$ be a Fano threefold with terminal Gorenstein singularities. Then $X$ is smoothable, that is, there exists a flat family $\mathfrak{X} \rightarrow \mathfrak{D} \ni 0$ over a small disc $(\mathfrak{D} \ni 0) \subset \mathbb{C}$ such that $\mathfrak{X}_{0} \simeq X$ and a general member $\mathfrak{X}_{s}, s \in \mathfrak{D}$ is a smooth Fano threefold. Moreover, there is a natural identification $\operatorname{Pic}(X)=\operatorname{Pic}\left(\mathfrak{X}_{s}\right)=\operatorname{Pic}(\mathfrak{X})$ so that $K_{\mathfrak{X}_{s}}=K_{X}($ see [JR06b, §1]). 
6.2. Now let $X$ be a Fano threefold with terminal Gorenstein singularities and let $\mathfrak{X} \rightarrow \mathfrak{D} \ni 0$ be its smoothing as above. We say that a reduced irreducible surface $S \subset X$ is a plane if $S \simeq \mathbb{P}^{2}$ and $\mathscr{O}_{S}\left(-K_{X}\right)=\mathscr{O}_{\mathbb{P}^{2}}(1)$. We say that a plane $S \subset X$ is contractible if there exists a birational morphism $f: X \rightarrow Y$ to a normal variety $Y$ such that $\rho(X / Y)=1, S$ is contained into the exceptional locus $\operatorname{Exc}(f)$ and $S$ does not meet other components of $\operatorname{Exc}(f)$ (if there is any). Note that, in this situation, $\operatorname{Exc}(f)$ is of pure dimension 2 (cf. [Kac98, Prop. 1.4]).

Recall that the nef cone $\operatorname{Nef}(X) \subset H^{2}(X, \mathbb{R})$ is the closed cone generated by nef divisors.

6.3. Proposition. Assume that, in the above notation, $X$ does not contain a contractible plane. Then under our identification $\operatorname{Pic}(X)=$ $\operatorname{Pic}(\mathfrak{X} / \mathfrak{D})=\operatorname{Pic}\left(\mathfrak{X}_{s}\right), s \in \mathfrak{D}$ we have $\operatorname{Nef}(X)=\operatorname{Nef}(\mathfrak{X} / \mathfrak{D})=\operatorname{Nef}\left(\mathfrak{X}_{s}\right)$.

Proof. Note that $\mathfrak{X}$ has only isolated hypersurface singularities. Hence, by [Gro68, Exp. XI, Corollary 3.14] the variety $\mathfrak{X}$ is (locally) factorial. Let $\mathcal{M}$ be a divisor on $\mathfrak{X}$, let $M:=\left.\mathcal{M}\right|_{X}$, and $\mathcal{M}_{s}:=\left.\mathcal{M}\right|_{\mathfrak{X}_{s}}$. If $M$ is nef, then, obviously, so $\mathcal{M}_{s}$ is. Thus it is sufficient to show the inverse implication. So, we assume that $\mathcal{M}_{s}$ is nef for some $s \in \mathfrak{D}$ and $M$ is not nef. Then $\mathcal{M}$ is also not nef. Let

$$
\lambda_{0}:=\sup \left\{\lambda \in \mathbb{Q} \mid \lambda \mathcal{M}-K_{\mathfrak{X}} \text { is nef over } \mathfrak{D}\right\} .
$$

By the rationality theorem we have $\lambda_{0} \in \mathbb{Q}$ and there is an extremal ray $R$ on $\mathfrak{X} / \mathfrak{D}$ such that $\left(\lambda_{0} \mathcal{M}-K_{\mathfrak{X}}\right) \cdot R=0$ and $K_{\mathfrak{X}} \cdot R<0$. Clearly, $\lambda_{0} \mathcal{M}-K_{\mathfrak{X}}$ is ample on $\mathfrak{X}_{s}$. Therefore, the locus $\operatorname{Exc}(R)$ of the ray $R$ does not meet $\mathfrak{X}_{s}$, so $\operatorname{Exc}(R) \subset X$. In particular, this means that $R$ is a flipping extremal ray. Then by Kac98 the locus $\operatorname{Exc}(R)$ is a disjoint union of irreducible surfaces $S_{i}$ isomorphic to $\mathbb{P}^{2}$ and, moreover, $\mathscr{O}_{S_{i}}\left(-K_{\mathfrak{X}}\right)=\mathscr{O}_{\mathbb{P}^{2}}(1)$. Hence, $X$ contains a contractible plane, a contradiction.

6.3.1. Corollary. Notation as in 6.2. Assume that $X$ does not contain a contractible plane. Let $\mathfrak{f}_{s}: \mathfrak{X}_{s} \rightarrow \mathfrak{Z}_{s}$ be an extremal contraction. Then there exists an extremal contraction $\mathfrak{f}: \mathfrak{X} \rightarrow \mathfrak{Z}$ over $\mathfrak{D}$ such that the restriction $\left.\mathfrak{f}\right|_{\mathfrak{X}_{s}}$ coincides with $\mathfrak{f}_{s}$.

6.4. Proposition. Let $X$ be a G-Fano threefold. Then $X$ does not contain a contractible plane.

Proof. Assume the converse. Let $S \subset X$ be a contractible plane and let $f: X \rightarrow Y$ be its contraction as in 6.2. Let $A$ be an ample Cartier divisor on $Y$ and let $M:=f^{*} A$. Thus $M$ is a nef and big divisor on $X$ and $M$ is trivial on $S$. Now let $\left\{M_{i}\right\}$ and $\left\{S_{i, j}\right\}$ be $G$-orbits of $M$ and $S$, respectively, where the subscript index $i$ is chosen so that $M_{i}$ 
is trivial on $S_{i, j}$. Then $M_{i}$ defines a contraction $f_{i}: X \rightarrow Y_{i}$ as in 6.2 and $\operatorname{Exc}\left(f_{i}\right)=\cup_{j} S_{i, j}$. For each fixed $i$, the surfaces $S_{i, j}$ do not meet each other. Assume that the intersection $S_{i, j} \cap S_{i^{\prime}, j^{\prime}}$ contains a curve $C$ for some $i \neq i^{\prime}$. Then $M_{i} \cdot C=M_{i^{\prime}} \cdot C=0$. So, $C$ is contracted by $f_{i}$ and $f_{i^{\prime}}$. This contradicts $\rho\left(X / Y_{i}\right)=\rho\left(X / Y_{i^{\prime}}\right)=1$. Therefore, any two different planes from $\left\{S_{i, j}\right\}$ intersect each other by a set of dimension $\leq 0$. On the other hand, $\sum_{i, j} S_{i, j}$ is a Cartier divisor proportional to $-K_{X}$. In particular, $\sum_{i, j} S_{i, j}$ is connected and has only a local complete intersection singularities, a contradiction.

Let $X$ be a $G$-Fano threefold with $\rho(X)>1$ (and terminal Gorenstein singularities). According to Theorem 1.2 its smoothing is of one of the types (1.2.1) - (1.2.8). In this situation, we say that $X$ is of the corresponding type (1.2.1) - (1.2.8).

6.5. Theorem. Let $X$ be a G-Fano threefold with $\rho(X)>1$.

(i) If $X$ is of type (1.2.4) or (1.2.7), then $X$ is smooth.

(ii) If $X$ is singular, then $X$ has the same description as in the table of Theorem 1.2 .

(iii) If $X$ is of type (1.2.3) and $X$ is singular, then $X$ is the blowup of a quadratic cone in $\mathbb{P}^{4}$ with center a union of two conics that do not pass through the vertex and meet each other transversely.

Proof. (i) follows from [Pro10].

To prove (ii) we consider only the case (1.2.1). All other cases are similar (see 2.3 for the case (1.2.2)). Then, in notation of 6.2 and 6.3.1, $\rho(\mathfrak{X} / \mathfrak{D})=2$ and the nef cone $\operatorname{Nef}(\mathfrak{X} / \mathfrak{D})$ has two edges. Thus there are two extremal contractions $\mathfrak{f}_{i}: \mathfrak{X} \rightarrow \mathfrak{Z}_{i}, i=1,2$ over $\mathfrak{D} \ni 0$. Let $\mathcal{M}_{i}, i=1,2$ be (integral) nef divisors generating edges of $\operatorname{Nef}(\mathfrak{X} / \mathfrak{D})$. We can take $\mathcal{M}_{i}$ to be primitive elements of $\operatorname{Pic}(\mathfrak{X} / \mathfrak{D}) \simeq \mathbb{Z}^{\oplus 2}$. Thus $\mathcal{M}_{i}=\mathfrak{f}_{i}^{*} \mathcal{A}_{i}$, where $\mathcal{A}_{i}$ is an ample generator of $\operatorname{Pic}\left(\mathfrak{Z}_{i} / \mathfrak{D}\right) \simeq \mathbb{Z}$. By Corollary 6.3.1 the map $\mathfrak{f}_{i}$ induces an extremal contraction $\mathfrak{f}_{i, s}: \mathfrak{X}_{s} \rightarrow$ $\mathfrak{Z}_{i, s}$ on a each fiber. For a general fiber $\mathfrak{X}_{s}, s \neq 0$ we have $\mathfrak{Z}_{i, s} \simeq \mathbb{P}^{2}$. Let $f_{i}: X \rightarrow Z_{i}$ be the contraction induced on the central fiber $X=\mathfrak{X}_{0}$ and let $M_{i}:=\left.\mathcal{M}_{i}\right|_{X}$. Then $M_{i}=f_{i}^{*} A_{i}$, where $A_{i}$ is an ample divisor on $Z_{i}$. By (5.1.2) $M_{1}+M_{2}=-K_{X}$. Since $M_{i}^{3}=$ $\left(\mathcal{M}_{i} \mid \mathfrak{x}_{s}\right)^{3}=0, Z_{i}$ is a surface and $f_{i}$ is a generically conic bundle. Hence, $A_{i}^{2}=-\frac{1}{2} M_{i}^{2} \cdot K_{X}=-\left(\left.\mathcal{M}_{i}\right|_{\mathfrak{X}_{s}}\right)^{2} \cdot K_{\mathfrak{X}_{s}}=1$. By semicontinuity $\operatorname{dim} H^{0}\left(Z_{i}, A_{i}\right)=\operatorname{dim} H^{0}\left(X, M_{i}\right) \geq \operatorname{dim} H^{0}\left(\mathfrak{X}_{s},\left.\mathcal{M}_{i}\right|_{\mathfrak{X}_{s}}\right)=3$. Hence, by Fuj75] $Z_{i} \simeq \mathbb{P}^{2}$. The map $f=f_{1} \times f_{2}: X \rightarrow \mathbb{P}^{2} \times \mathbb{P}^{2}$ must be finite and $G$-equivariant. 
Let $\left(d_{1}, d_{2}\right)$ be the bidegree of $f(X)$ in $\mathbb{P}^{2} \times \mathbb{P}^{2}$ (as a divisor). Then

$$
d_{i}=\mathscr{O}_{\mathbb{P}^{2} \times \mathbb{P}^{2}}(1,0) \cdot \mathscr{O}_{\mathbb{P}^{2} \times \mathbb{P}^{2}}(1,0)^{2} \cdot f(X)=\frac{M_{1} \cdot M_{2}^{2}}{\operatorname{deg} f}=\frac{2}{\operatorname{deg} f} .
$$

Similarly, $d_{2}=2 / \operatorname{deg} f$. Thus $d_{1}=d_{2}$ and there are two possibilities:

a) $f(X)$ is of bidegree $(2,2)$ and $f$ is birational;

b) $f(X)$ is of bidegree $(1,1)$ and $f$ is finite of degree 2 .

In the first case, the map $f: X \rightarrow f(X)$ is birational and finite. By subadjunction $K_{X}=f^{*} K_{f(X)}-B$, where $B$ is an effective divisor. On the other hand, $K_{X}=-M_{1}-M_{2}=f^{*} \mathscr{O}_{\mathbb{P}^{2} \times \mathbb{P}^{2}}(-1,-1)=f^{*} K_{f(X)}$. Hence, $B=0, f(X)$ is normal, and $f: X \rightarrow f(X)$ is an isomorphism.

In the case $\mathrm{b})$, we note that any irreducible singular hypersurface of bidegree $(1,1)$ in $\mathbb{P}^{2} \times \mathbb{P}^{2}$ can be given, in some coordinate system, by the equation $x_{1} y_{1}+x_{2} y_{2}=0$. In this case, the singular locus of $f(X)$ consists of one node and $f(X)$ contains exactly two planes. Therefore, $\operatorname{rk~} \mathrm{Cl}(f(X))^{G}>1$ and so $\operatorname{rk} \mathrm{Cl}(X)>1$, a contradiction. Hence $f(X)$ is smooth.

Now we prove (iii). As above, by Corollary 6.3.1 two extremal contractions $\mathfrak{f}_{i}: \mathfrak{X} \rightarrow \mathfrak{Z}_{i}, i=1,2$ over $\mathfrak{D} \ni 0$ induce extremal contractions $\mathfrak{f}_{i, s}: \mathfrak{X}_{s} \rightarrow \mathfrak{Z}_{i, s}$ on a each fiber and for $s \neq 0$ the variety $\mathfrak{Z}_{i, s}$ is a smooth quadric. Hence $\mathfrak{f}_{i}$ induces a birational extremal contraction $f_{i}: X \rightarrow Q_{i}$ on the central fiber $X=\mathfrak{X}_{0}$. Let $\mathcal{M}_{i}=\mathfrak{f}_{i}^{*} \mathcal{A}_{i}$, where $\mathcal{A}_{i}$ is an ample generator of $\operatorname{Pic}\left(\mathfrak{Z}_{i} / \mathfrak{D}\right) \simeq \mathbb{Z}$ and let $M_{i}:=\left.\mathcal{M}_{i}\right|_{X}$. Then $M_{i}=f_{i}^{*} A_{i}$, where $A_{i}$ is an ample divisor on $Z_{i}$. By semicontinuity $\operatorname{dim} H^{0}\left(Q_{i}, A_{i}\right) \geq 5$. Hence, by [Fuj75] $Q_{i}$ is a quadric in $\mathbb{P}^{4}$. In particular, $K_{Q_{i}}$ is Cartier. Since $-K_{X}$ is ample, by standard facts of the minimal model program $Q_{i}$ has at worst terminal singularities. Moreover, $f_{i}$ is an isomorphism over the singular locus of $Q_{i}$. Hence $Q_{i}$ is either a smooth quadric or a quadratic cone in $\mathbb{P}^{4}$. Let $\mathcal{E}_{i} \subset \mathfrak{X}$ be $\mathfrak{f}_{i}$-exceptional divisor. Since $\mathfrak{X}$ is factorial, $\mathcal{E}_{i}$ is an irreducible Cartier divisor. Let $E_{i}:=\left.\mathcal{E}_{i}\right|_{X}$. Clearly, the divisor $E_{i}$ coincides with the $f_{i}$-exceptional locus. We have $-K_{X}=M_{1}+M_{2}=E_{1}+E_{2}$ (because this holds on a general fiber).

Let $G_{\bullet} \subset G$ be the subgroup of index 2 that stabilizes $M_{1}$ (and $\left.M_{2}\right)$. Then the contraction $f_{i}$ is $G_{\bullet}$-equivariant. Since $\operatorname{rk} \operatorname{Cl}\left(Q_{i}\right) \leq 2$, we have $\operatorname{Cl}\left(Q_{i}\right)^{G_{\bullet}} \simeq \mathbb{Z}$.

Write $E_{i}=\sum_{j=1}^{r} k_{i} E_{i}^{(j)}$, where $k_{i}>0, r \geq 1$, and $E_{i}^{(j)}$ are prime Weil divisors. Let $\Gamma_{i}:=f_{i}\left(E_{i}\right)$ and let $\Gamma_{i}^{(j)}=f_{i}\left(E_{i}^{(j)}\right)$. Since $\operatorname{Cl}(X)^{G}$ is generated by $K_{X}$, the divisors $E_{i}^{(j)}$ form one $G$-orbit and $k_{i}=1$ for all $i$. In particular, $E_{1}$ and $E_{2}$ have no common components and $\Gamma_{i}^{(j)}$ is a curve for all $j$ (because $E_{1} \neq E_{2}$ and $E_{1} \cdot E_{2} \cdot M_{i} \neq 0$ ). Let $D \in\left|-K_{X}\right|$ be a general member and let $D_{i}:=f_{i}(D)$. Then $D_{i} \in\left|-K_{Q_{i}}\right|$. Since 
the linear system $\left|-K_{X}\right|$ is base point free [JR06a], $D$ is a smooth K3 surface. We may take $S$ so that it does not contain non-trivial fibers of $E_{i} \rightarrow \Gamma_{i}$ and meets a general fiber transversely at one point. Then $D \rightarrow D_{i}$ is a finite birational morphism and $D_{i}$ has at worst isolated singularities. Since $D_{i}$ is Cohen-Macaulay, it is smooth and $D \rightarrow D_{i}$ is an isomorphism. Then

$$
\begin{aligned}
& \operatorname{deg} \Gamma_{i}=A_{i} \cdot \Gamma_{i}=-K_{X} \cdot M_{i} \cdot E_{i}=4 \\
& -2=-K_{X} \cdot E_{i}^{2}=D \cdot E_{i}^{2}=\Gamma_{i}^{2}=2 p_{a}\left(\Gamma_{i}\right)-2 \quad \Longrightarrow \quad p_{a}\left(\Gamma_{i}\right)=0 .
\end{aligned}
$$

Since $\left(-K_{X}\right)^{2} \cdot E_{1}=\frac{1}{2}\left(-K_{X}\right)^{3}=14, r$ divides 14. Further, since $-K_{X} \cdot M_{1} \cdot E_{1}=4, r$ divides 4 .

Therefore, $r=1$ or 2 . If $r=1$, i.e., $E_{i}$ is irreducible, then so $\Gamma_{i}:=f_{i}\left(E_{i}\right)$ is. Thus $\Gamma_{i}$ is a smooth rational curve of degree 4 . In this case, $\Gamma_{i}$ is contained into the smooth locus of $Q_{i}, f_{i}$ is the blowup of $\Gamma_{i}$, and $X$ is smooth along $E_{i}$ Cut88. Assume that $Q_{i}$ is singular, i.e., it is the projective cone over a smooth quadric $W \simeq \mathbb{P}^{1} \times \mathbb{P}^{1}$ in $\mathbb{P}^{3}$. Let $O_{i} \in Q_{i}$ be the vertex of the cone. Then $X$ has a unique singular point $O \in X$ and $f_{i}(O)=O_{i}$. Moreover, $O \notin E_{i}$. Since $E_{1}+E_{2} \sim-K_{X}$, $f_{1}\left(E_{2}\right) \sim-K_{Q_{1}}$. Moreover, $f_{1}\left(E_{2}\right)$ is singular along $\Gamma_{1}$. Hence every 2 -secant line to $\Gamma_{1}$ is contained in $f_{1}\left(E_{2}\right)$. Since $O_{1} \notin f_{1}\left(E_{2}\right)$, the projection of $\Gamma_{1}$ from $O_{1}$ to $W$ is an isomorphism. Thus $\Gamma_{1}$ is contained into a divisor of type $(1,3)$ on $Q_{1}$. But in this case, the action of $G$. on $\operatorname{Cl}\left(Q_{i}\right) \simeq \mathbb{Z} \oplus \mathbb{Z}$ must be trivial, a contradiction.

Finally, assume that $r=2$, i.e., $E_{i}=E_{i}^{(1)}+E_{i}^{(2)}$. Since the divisors $E_{i}^{(1)}$ and $E_{i}^{(2)}$ are not Cartier, $G_{\bullet}$ interchanges $E_{i}^{(1)}$ and $E_{i}^{(2)}$. Hence, $G_{\bullet}$ interchanges also $f_{1}\left(E_{2}^{(1)}\right)$ and $f_{1}\left(E_{2}^{(2)}\right)$. On the other hand, $f_{1}\left(E_{2}^{(1)}\right)+f_{1}\left(E_{2}^{(2)}\right) \sim-K_{Q_{1}} \sim \mathscr{O}_{Q_{1}}(3)$. This is possible only if $f_{1}\left(E_{2}^{(1)}\right)$ and $f_{1}\left(E_{2}^{(2)}\right)$ are not Cartier. So, $Q_{1}$ is the quadratic cone and $f_{1}\left(E_{2}\right)$ contains its vertex. Again by Cut88] $X$ is the blowup of $Q_{1}$ along $\Gamma_{1}$.

\section{REFERENCES}

[AB92] A. Alzati and M. Bertolini. On the rationality of Fano 3-folds with $B_{2} \geq$ 2. Matematiche (Catania), 47(1):63-74, 1992.

[CK89] B. Crauder and S. Katz. Cremona transformations with smooth irreducible fundamental locus. Amer. J. Math., 111(2):289-307, 1989.

[Cut88] S. Cutkosky. Elementary contractions of Gorenstein threefolds. Math. Ann., 280(3):521-525, 1988.

[Fuj75] T. Fujita. On the structure of polarized varieties with $\Delta$-genera zero. $J$. Fac. Sci. Univ. Tokyo Sect. IA Math., 22:103-115, 1975. 
[Gro68] A. Grothendieck. Cohomologie locale des faisceaux cohérents et théorèmes de Lefschetz locaux et globaux (SGA 2). North-Holland Publishing Co., Amsterdam, 1968. Augmenté d'un exposé par Michèle Raynaud, Séminaire de Géométrie Algébrique du Bois-Marie, 1962, Advanced Studies in Pure Mathematics, Vol. 2.

[JR06a] P. Jahnke and I. Radloff. Gorenstein Fano threefolds with base points in the anticanonical system. Compos. Math., 142(2):422-432, 2006.

[JR06b] P. Jahnke and I. Radloff. Terminal Fano threefolds and their smoothings. Math. Z. 269(3-4): 1129-1136, 2011.

[Kac98] Y. Kachi. Flips from 4-folds with isolated complete intersection singularities. Amer. J. Math., 120(1):43-102, 1998.

[Kat87] S. Katz. The cubo-cubic transformation of $\mathbf{P}^{3}$ is very special. Math. Z., 195(2):255-257, 1987.

[MM83] S. Mori and S. Mukai. On Fano 3-folds with $B_{2} \geq 2$. In Algebraic varieties and analytic varieties (Tokyo, 1981), volume 1 of Adv. Stud. Pure Math., pages 101-129. North-Holland, Amsterdam, 1983.

[MM86] S. Mori and S. Mukai. Classifications of Fano 3-folds with $B_{2} \geq 2$, I. In Algebraic and topological theories. Papers from the symposium dedicated to the memory of Dr. Takehiko Miyata held in Kinosaki, October $30-$ November 9, 1984, pages 496-545. Kinokuniya, Tokyo, 1986.

[MM82] S. Mori and S. Mukai. Classification of Fano 3-folds with $B_{2} \geq 2$. Manuscripta Math., 36(2):147-162, 1981/82. Erratum: Manuscripta Math. 110 (2003), 407.

[Nak89] T. Nakano. On equivariant completions of 3-dimensional homogeneous spaces of SL(2, C). Japan. J. Math. (N.S.), 15(2):221-273, 1989.

[Nam97] Y. Namikawa. Smoothing Fano 3-folds. J. Algebraic Geom., 6(2):307324, 1997.

[Pro09] Y. Prokhorov. Simple finite subgroups of the Cremona group of rank 3. J. Algebraic Geom. 21(3): 563-600, 2012.

[Pro10] Y. Prokhorov. G-Fano threefolds, I. ArXiv e-prints, (arXiv:1012.4959), 2010.

[Pro10b] Yu. Prokhorov. p-elementary subgroups of the Cremona group of rank 3. In C. Faber, G. van der Geer, and E. Looijenga, editors, Classification of Algebraic Varieties, Schiermonnikoog, 2009, 327-338. European Math. Soc., 2010.

[Ser07] J.-P. Serre. Bounds for the orders of the finite subgroups of $G(k)$. In Group representation theory, pages 405-450. EPFL Press, Lausanne, 2007.

Department of Algebra, Faculty of Mathematics, Moscow State UnIVERSITY, Moscow, 119 991, RUSSIA

Laboratory of Algebraic Geometry, SU-HSE, 7 Vavilova Str., Moscow, 117312, RUSSIA

E-mail address: prokhoro@gmail.com 